\title{
Methods for the Numerical Solution of the Nonlinear Schroedinger Equation
}

\author{
By J. M. Sanz-Serna
}

\begin{abstract}
Optimal $L^{2}$ rates of convergence are established for several fully-discrete schemes for the numerical solution of the nonlinear Schroedinger equation. Both finite differences and finite elements are considered for the discretization in space, while the integration in time is treated either by the leap-frog technique or by a modified Crank-Nicolson procedure, which generalizes the one suggested by Delfour, Fortin and Payne and possesses two useful conserved quantities.
\end{abstract}

1. Introduction. The nonlinear Schroedinger equation

$$
i u_{t}+\Delta u+\lambda|u|^{p-1} u=0, \quad i^{2}=-1, p>1,
$$

has been used extensively to model nonlinear dispersive waves [10]. Here $u$ is complex, $u=u(\mathbf{x}, t), \mathbf{x} \in R^{n}, t>0$ and $\lambda$ is a real constant. The pure initial value problem and the periodic value problem for (1.1) have the invariants of motion

$$
\begin{gathered}
E(u)=\int|u|^{2} d \mathbf{x}, \\
I(u)=\int \frac{1}{2}\left[|\nabla u|^{2}-(\lambda /(p+1))|u|^{p+1}\right] d \mathbf{x},
\end{gathered}
$$

which plays an important role in the analysis of the equation. A survey of results is provided by Strauss [10]. We recall that the regularity and existence for all positive $t$ of the solutions depend on $p, n$ and the sign of $\lambda$. The conservation of the $L^{2}$ norm guaranteed by (1.2.a) is not strong enough to ensure that the solutions are defined for all positive $t$. In fact, if $\lambda>0, I(u(\cdot, 0))<0$ and $p \geqslant 1+4 / n$, then no smooth solution can exist for all positive $t$. On the other hand, if $\lambda<0$, (1.2.b) provides trivially a priori bounds for the $H^{1}$ and $L^{p+1}$ norms, and use of the Sobolev inequality [10] reveals that those bounds also exist when $\lambda>0$, provided that $p<1+4 / n$.

Zakharov and Shabat [14] developed an inverse scattering procedure for the initial value problem for (1.1), in the highly important particular case $p=3, n=1, \lambda>0$. It is useful to point out that in this case, the constant modulus, $x$-independent solution $b \exp \left(i \lambda|b|^{2} t\right)$ is "unstable" to 'infinitesimal' long-wave perturbations [13] (i.e. the linearization around that solution exhibits growing Fourier modes). This

Received September 24, 1982; revised April 19, 1983, June 13, 1983, and October 13, 1983.

1980 Mathematics Subject Classification. Primary 65M10. 
phenomenon is related to the Benjamin-Feir [2] instability in the theory of water waves. Initially, the 'unstable' Fourier modes draw energy from the lower modes, but due to (1.2.a) this process cannot continue indefinitely, and in fact it is possible for the energy to return to its initial distribution among the Fourier modes after a period of time (Fermi-Pasta-Ulam recurrence [13]).

A number of finite-difference and finite-element schemes have been suggested for the numerical study of (1.1). Delfour, Fortin and Payne [3] propose a modified Crank-Nicolson technique which conserves both (1.2.a) and (1.2.b) when finite elements are used for the discretization in space. (If finite differences in space are employed, the scheme conserves the discrete analogues of (1.2.a,b).) Griffiths, Mitchell and Morris [5] study predictor-corrector schemes. Sanz-Serna and Manoranjan [9] consider the leap-frog technique in time and also suggest a variablestep modification whereby (1.2.a) can be conserved. The importance of the numerical conservation of the invariants of motion in the numerical study of partial differential equations describing wave phenomena cannot be overemphasized. In particular, it is well-known that failure in the conservation of (1.2.a) can lead to blow-up of the computed solution, and this is particularly so in the present instance due to the role played by the conservation laws in the dynamics of the solutions [5], [9]. Additional references for (1.1) and its numerical solution can be found in [3], [5], [10], [13].

In the present paper the energy method will be used in a standard way (cf. [4], [8], [12]) to study the convergence of the schemes cited above. For brevity we only present the proofs in the cases of a leap-frog finite-difference method and a modified Crank-Nicolson finite-element procedure which generalizes that of Delfour, Fortin and Payne.

We consider the one-dimensional problem

$$
\begin{aligned}
& i u_{t}+u_{x x}+a\left(|u|^{2}\right) u=0, \\
& u(x, t)=u(x+1, t), \quad x \in \mathbf{R}, t>0 \\
& u(x, 0)=u_{0}(x)
\end{aligned}
$$

(with $u_{0}$ a given 1-periodic function), as the extension to problems with $n=2,3$ is easy. In (1.3), $a(\cdot)$ denotes a continuous real function of real argument, such that the composition $z \rightarrow a^{*}(z)=a\left(|z|^{2}\right)$ has continuous partial derivatives $\partial a^{*} / \partial(\operatorname{Re} z)$, $\partial a^{*} / \partial(\operatorname{Im} z)$ with respect to the real and imaginary parts of $z$. Thus the nonlinearities $|u|^{p-1} u$ are included in (1.3) provided that $p \geqslant 2$.

The problem has the conserved quantities (1.2.a) and

$$
I(u)=\int \frac{1}{2}\left[\left|u_{x}\right|^{2}-A\left(|u|^{2}\right)\right] d x
$$

where $A(\cdot)$ is a primitive function of $a(\cdot)$. These invariants of motion are derived by taking the imaginary part of the inner product of (1.3) and $u$, and by taking the real part of the inner product of (1.3) and $u_{t}$, respectively.

Throughout the paper we assume that the initial condition and the function $a(\cdot)$ are such that (1.3) has a unique smooth solution $u$ defined in $0 \leqslant t \leqslant T$; cf. [10]. More precise smoothness assumptions are given later. 
2. A Leap-Frog Scheme. We introduce a grid $x_{j}=j h, j=0, \pm 1, \pm 2, \ldots, h=1 / J$, $J$ a positive integer. If $V, W$ are 1-periodic grid functions, we denote by $V_{j}, W_{j}$ their values at $x_{j}$ and set

$$
\begin{gathered}
(V, W)=h \sum_{j=1}^{J} V_{j} \bar{W}_{j}, \\
\|V\|^{2}=(V, V), \\
\delta^{2} V_{j}=h^{-2}\left[V_{j+1}-2 V_{j}+V_{j-1}\right] .
\end{gathered}
$$

We shall need the identity

$$
\left(V, \delta^{2} W\right)=\left(\delta^{2} V, W\right)
$$

and the bound

$$
\left\|\delta^{2} W\right\| \leqslant 4 h^{-2}\|W\| .
$$

The step length in time is denoted by $k$ and a superscript $n$ refers to the time level $t_{n}=n k$. We set

$$
D V_{j}^{n}=(1 / 2 k)\left(V_{j}^{n+1}-V_{j}^{n-1}\right),
$$

if $V^{n}, n=0,1, \ldots, N$, is a family of grid functions and $N=[T / k]$.

With this notation we are in a position to consider the method

$$
i D U_{j}^{n}+\delta^{2} U_{j}^{n}+a\left(\left|U_{j}^{n}\right|^{2}\right) U_{j}^{n}=0, \quad j=1, \ldots, J ; n=1, \ldots, N-1 ;
$$

for which the following result holds:

Theorem 1. Assume that $u \in C^{4}$ and that $\left\|U^{0}-u^{0}\right\|+\left\|U^{1}-u^{1}\right\|=o\left(h^{1 / 2}\right)$. If there exists a constant $\sigma$ with $k h^{-2} \leqslant \sigma<1 / 4$, then there exist positive constants $C_{m}$, $m=1,2,3$, independent of $k$ and $h$ such that, for $k \leqslant C_{1}, h \leqslant C_{2}$,

(2.8) $\left\|U^{n}-u^{n}\right\| \leqslant C_{3}\left(k^{2}+h^{2}+\left\|U^{0}-u^{0}\right\|+\left\|U^{1}-u^{1}\right\|\right), \quad n=0,1, \ldots, N$.

Proof. We set $e^{n}=U^{n}-u^{n}$ and

$$
B^{n}=\left\|e^{n}\right\|^{2}+\left\|e^{n-1}\right\|^{2}+2 k \operatorname{Im}\left(\delta^{2} e^{n-1}, e^{n}\right) .
$$

Use of (2.5) leads to the bounds

$$
(1-4 \sigma)\left(\left\|e^{n}\right\|^{2}+\left\|e^{n-1}\right\|^{2}\right) \leqslant B^{n} \leqslant(1+4 \sigma)\left(\left\|e^{n}\right\|^{2}+\left\|e^{n-1}\right\|^{2}\right),
$$

so that $B^{n}$ is a positive definite function of $e^{n}, e^{n-1}$.

The truncation error defined by

$$
\tau_{j}^{n}=i D u_{j}^{n}+\delta^{2} u_{j}^{n}+a\left(\left|u_{j}^{n}\right|^{2}\right) u_{j}^{n} ; \quad j=1, \ldots, J ; n=1, \ldots, N-1
$$

is easily seen to be $O\left(k^{2}+h^{2}\right)$.

Subtraction of (2.11) from (2.7), multiplication by $\bar{e}_{j}^{n+1}+\bar{e}_{j}^{n-1}$, summation and consideration of the imaginary part yield

$$
\begin{aligned}
(1 / 2 k)\left(B^{n+1}-B^{n}\right)= & -\operatorname{Im}\left(a\left(\left|U^{n}\right|^{2}\right) U^{n}-a\left(\left|u^{n}\right|^{2}\right) u^{n}, e^{n+1}+e^{n-1}\right) \\
& +\operatorname{Im}\left(\tau^{n}, e^{n+1}+e^{n-1}\right) .
\end{aligned}
$$


Here we have used the identity (2.4) and the definition of $B^{n}$. We now make the additional hypothesis that $a^{*}, \partial a^{*} / \partial(\operatorname{Re} z), \partial a^{*} / \partial(\operatorname{Im} z)$ are bounded. Then $(2.12)$ implies

$$
\left\|B^{n+1}-B^{n}\right\| \leqslant k\left\{\left\|\tau^{n}\right\|^{2}+2\left\|e^{n+1}+e^{n-1}\right\|^{2}+K\left\|e^{n}\right\|^{2}\right\}
$$

where $K$ is a constant independent of $k, h$. The second inequality in (2.10) and Gronwall's lemma show that (2.8) holds for arbitrary $k, h$ satisfying $k h^{-2} \leqslant \sigma$.

In order to remove the additional hypothesis on boundedness, we resort to a standard argument (see [1] among others). A function $\hat{a}$ is introduced which is bounded and possesses bounded derivatives and which agrees with $a$ in a neighborhood of $\{u(x, t): 0 \leqslant x \leqslant 1,0 \leqslant t \leqslant T\}$. An easy energy estimate shows that for $\hat{a}$ the problem (1.3) has at most one solution and therefore has no solution other than $u$. From the result above we conclude that when $a$ is replaced by $\hat{a}$, the discrete solutions satisfy (2.8) and in particular converge uniformly. But then for $h, k$ small $\hat{a}\left(\left|U^{n}\right|^{2}\right)=a\left(\left|U^{n}\right|^{2}\right)$ and the proof is complete.

Remark 1 . An elementary computation shows that $k h^{-2} \leqslant 1 / 4$ is the von Neumann condition for the equation $i u_{t}+u_{x x}=0$.

Remark 2. The leap-frog scheme is very easy to code and rather cheap to run. However, numerical experiments reported in [9] reveal that it is subject to the occurrence of numerical blow-up. According to the theorem, the blow-up can be delayed by reducing $k$ and $h$ (see the discussion in [6]). A variable-step modification which conserves the discrete $L^{2}$ norm of the solution is studied in [9]. Of course the blow-up can also be prevented by the introduction of artificial viscosity.

Remark 3. The missing starting level $U^{1}$ can be obtained by means of a one-step scheme based on the replacement of $u_{t}$ by forward differences. (See [9] for details.)

3. A Modified Crank-Nicolson Scheme. In this section $\|\cdot\|,($, ) represent the usual norm and inner product in the complex space $L^{2}(0,1),\|\cdot\|_{\infty}$ the supremum norm in $L^{\infty}(0,1)$. We consider the periodic Sobolev space $H_{p}^{1}$ and for each of a family of values of $h$ in $(0,1)$ clustering at 0 denote by $S_{h}$ a finite-dimensional subspace of $H_{p}^{1}$.

An inverse assumption,

$$
\sup \left\{h^{1 / 2}\|\phi\|_{\infty} /\|\phi\|: \phi \in S_{h}, \phi \neq 0\right\}=\alpha<\infty
$$

(with $\alpha$ independent of $h$ ), is required.

For each fixed value of $t$, let the $H^{1}$-projection $W(t)$ of $u(t)$ onto $S_{h}$ be defined by the relations $W(t) \in S_{h}$,

$$
\left(u_{x}(t)-W_{x}(t), \phi_{x}\right)+(u(t)-W(t), \phi)=0 \text { for all } \phi \text { in } S_{h},
$$

and assume that the following approximation property holds:

There exists a positive integer $r$ and a positive constant $C$, independent of $h$, such that

$$
\sup _{0 \leqslant t \leqslant T}\left\{\|u-W\|_{\infty}+\left\|u_{t}-W_{t}\right\|\right\} \leqslant C h^{r}
$$

We also assume that $u_{t t t}$ is continuous. 
As in the previous section $k$ denotes the step size in time, $N=[T / k]$ and a superscript $n$ refers to the level $t_{n}=n k$. Furthermore we set

$$
\begin{aligned}
D_{+} v^{n} & =k^{-1}\left(v^{n+1}-v^{n}\right), \\
v^{n+1 / 2} & =(1 / 2)\left(v^{n+1}+v^{n}\right),
\end{aligned}
$$

if $v^{n}, n=0,1, \ldots, N$, is a family of functions indexed by $n$.

It is also convenient to introduce a real function of two complex variables as follows:

$$
F\left(z_{1}, z_{2}\right)=\left[A\left(\left|z_{1}\right|^{2}\right)-A\left(\left|z_{2}\right|^{2}\right)\right] /\left[\left|z_{1}\right|^{2}-\left|z_{2}\right|^{2}\right]
$$

if $\left|z_{1}\right| \neq\left|z_{2}\right|$ and

$$
F\left(z_{1}, z_{2}\right)=a\left(\left|z_{1}\right|^{2}\right)
$$

otherwise. From the mean value theorem and the properties of $a(\cdot)$ we conclude that $F$ is a continuous function and that $F\left(z_{1}, z_{2}\right)=a\left(\left|\left(z_{1}+z_{2}\right) / 2\right|^{2}\right)+O\left(\left|z_{1}-z_{2}\right|^{2}\right)$.

The modified Crank-Nicholson-Galerkin solution $U^{n} \in S_{h}, n=0,1, \ldots, N$, is defined by

$$
i\left(D_{+} U^{n}, \phi\right)-\left(U_{x}^{n+1 / 2}, \phi_{x}\right)+\left(F\left(U^{n+1}, U^{n}\right) U^{n+1 / 2}, \phi\right)=0
$$

for all $\phi \in S_{h}$, with $u_{0}-U^{0}$ 'small'.

The method contains as a particular case the Delfour, Fortin and Payne scheme [3], whose main idea goes back to Strauss and Vazquez [11]. The choices $\phi=U^{n+1 / 2}$, $\phi=D_{+} U^{n}$ reveal that (3.7) conserves both (1.2.a) and (1.4). Therefore the computed solution possesses an a priori $L^{2}$ bound (and according to the introduction, an a priori $H^{1}$ bound in some important particular cases). Note that the evaluation of $F$ is essentially a numerical differentiation and may cause trouble due to cancellations in the denominator of (3.5). However, if $a(\cdot)$ is a polynomial, it is possible to write the right-hand side of (3.5) as a polynomial in $\left|z_{1}\right|^{2},\left|z_{2}\right|^{2}$ thus avoiding the danger of cancellations. For instance for the nonlinearity $|u|^{2} u$ (i.e. $a(y)=y$ ), $F$ becomes $\frac{1}{2}\left[\left|z_{1}\right|^{2}+\left|z_{2}\right|^{2}\right]$.

TheOREM 2. Assume that as $h \rightarrow 0,\left\|U^{0}-u_{0}\right\|=o\left(h^{1 / 2}\right), k=o(h)$. Then there exist positive constants $C_{m}, m=1,2$, independent of $k$ and $h$, such that if $h \leqslant C_{1}$, (3.7) has a solution with

$$
\left\|U^{n}-u^{n}\right\| \leqslant C_{2}\left(k^{2}+h^{r}+\left\|U^{0}-u_{0}\right\|\right), \quad n=0,1, \ldots, N .
$$

Proof. A (Browder) fixed point argument shows the existence of solutions. We average the differential equation written in weak form at time levels $n$ and $n+1$ to obtain

$$
i\left(D_{+} u^{n}, \phi\right)-\left(u_{x}^{n+1 / 2}, \phi_{x}\right)+\left(F\left(u^{n+1}, u^{n}\right) u^{n+1 / 2}, \phi\right)=\left(\rho^{n}, \phi\right),
$$

where $k \sum_{n=0}^{N-1}\left\|\rho^{n}\right\|^{2} \leqslant C k^{4}$, with $C$ independent of $k$. Now define $e=U-u, \eta=W$ $-u, \zeta=U-W$, and use (3.1) (3.7), (3.8) to write

$$
\text { (3.10) } \begin{aligned}
i\left(D_{+} \zeta^{n}, \phi\right)= & -i\left(D_{+} \eta^{n}, \phi\right)+\left(\zeta_{x}^{n+1 / 2}, \phi_{x}\right)+\left(\eta^{n+1 / 2}, \phi\right) \\
& -\left(F\left(U^{n+1}, U^{n}\right) U^{n+1 / 2}-F\left(u^{n+1}, u^{n}\right) u^{n+1 / 2}, \phi\right)-\left(\rho^{n}, \phi\right) .
\end{aligned}
$$


Set $\phi=\zeta^{n+1 / 2}$, note that $\left(\zeta_{x}, \zeta_{x}\right)$ is real and take imaginary parts to arrive at

$$
\begin{aligned}
D_{+}\left\|\zeta^{n}\right\|^{2} \leqslant & 4\left\|\zeta^{n+1 / 2}\right\|^{2}+\left\|D_{+} \eta^{n}\right\|^{2}+\left\|\eta^{n+1 / 2}\right\|^{2}+\left\|\rho^{n}\right\|^{2} \\
& +\left\|F\left(U^{n+1}, U^{n}\right) U^{n+1 / 2}-F\left(u^{n+1}, u^{n}\right) u^{n+1 / 2}\right\|^{2} .
\end{aligned}
$$

Recall that due to the conserved quantities the norms $\left\|\zeta^{n}\right\|$ have an a priori bound. We now inductively assume that $\left\|\zeta^{m}\right\|_{\infty} \leqslant 1, m=0,1, \ldots, n$.

The only difficulty in (3.11) arises from the nonlinear term. With $K$ a constant independent of $k$ and $h$, whose value is not necessarily the same at each occurrence, we may write

$$
\begin{aligned}
&\left\|F\left(U^{n+1}, U^{n}\right) U^{n+1 / 2}-F\left(u^{n+1}, u^{n}\right) u^{n+1 / 2}\right\| \\
& \leqslant\left\|F\left(U^{n+1}, U^{n}\right)\left(U^{n+1 / 2}-u^{n+1 / 2}\right)\right\| \\
&+\left\|\left(F\left(U^{n+1}, U^{n}\right)-F\left(u^{n+1}, u^{n}\right)\right) u^{n+1 / 2}\right\| \\
& \leqslant K\left(\left\|e^{n+1 / 2}\right\|+\left\|F\left(U^{n+1}, U^{n}\right)-F\left(u^{n+1}, u^{n}\right)\right\|\right) .
\end{aligned}
$$

Now

$$
\begin{aligned}
\left\|F\left(U^{n+1}, U^{n}\right)-F\left(u^{n+1}, u^{n}\right)\right\| & =\left\|a^{*}\left(U^{n+1 / 2}\right)-a^{*}\left(u^{n+1 / 2}\right)\right\|+\left\|Y_{n}\right\| \\
& \leqslant K\left\|e^{n+1 / 2}\right\|+\left\|Y_{n}\right\|
\end{aligned}
$$

where we have set

$$
Y_{n}=F\left(U^{n+1}, U^{n}\right)-F\left(u^{n+1}, u^{n}\right)-a^{*}\left(U^{n+1 / 2}\right)+a^{*}\left(u^{n+1 / 2}\right),
$$

and we have assumed that $a^{*}$ has bounded derivatives. (This implies no loss of generality: we may resort to the argument used in the proof of Theorem 1.)

The mean value theorem yields, pointwise,

so that,

$$
\begin{aligned}
\left|Y_{n}\right| & \leqslant K\left(\left|U^{n+1}-U^{n}\right|^{2}+\left|u^{n+1}-u^{n}\right|^{2}\right) \\
& \leqslant K\left(\left|\zeta^{n+1}\right|^{2}+\left|\zeta^{n}\right|^{2}+\left|\eta^{n+1}\right|^{2}+\left|\eta^{n}\right|^{2}+\left|u^{n+1}-u^{n}\right|^{2}\right),
\end{aligned}
$$

$$
\begin{aligned}
\left\|Y_{n}\right\| \leqslant K\left(\left\|\zeta^{n+1}\right\|\left\|\zeta^{n+1}\right\|_{\infty}+\left\|\zeta^{n}\right\|\left\|\zeta^{n}\right\|_{\infty}+\left\|\eta^{n+1}\right\|_{\infty}^{2}\right. & \\
& \left.+\left\|\eta^{n}\right\|_{\infty}^{2}+\left\|u^{n+1}-u^{n}\right\|_{\infty}^{2}\right) .
\end{aligned}
$$

If $\left\|\zeta^{n+1}\right\|_{\infty},\left\|\zeta^{n}\right\|_{\infty} \leqslant 1$, then (3.14) yields

$$
\left\|Y_{n}\right\| \leqslant K\left(\left\|\zeta^{n+1}\right\|+\left\|\zeta^{n}\right\|+h^{2 r}+k^{2}\right)
$$

which upon substitution in (3.11) gives (3.8) via Gronwall's lemma. In order to conclude the proof we must show that for $k, h$ small $\left\|\zeta^{m}\right\|_{\infty} \leqslant 1, m=0,1, \ldots$. Assume this to be true for $m \leqslant n$. Then (3.14) and the inverse inequality show

$$
\left\|Y_{n}\right\| \leqslant K\left(\left\|\zeta^{n}\right\|+h^{-1 / 2}\left\|\zeta^{n+1}\right\|+h^{2 r}+k^{2}\right)
$$

Substitution in (3.11) proves that

$$
\left(1-K k h^{-1}\right)\left\|\zeta^{n+1}\right\|^{2} \leqslant(1+K k)\left\|\zeta^{n}\right\|^{2}+k K\left(h^{2 r}+k^{4}\right) .
$$

Now (3.8) applies up to $t=n k$ and one sees without difficulty that for $k, h$ small $\left\|\zeta^{n+1}\right\|_{\infty} \leqslant 1$.

Remark. In the cases where conservation of (1.4) leads to a priori $H^{1}$ and $L^{\infty}$ bounds the inductional hypothesis of the proof can be dispensed with. 
4. Concluding Remarks. The study of the finite-difference analogue of the method in Section 3 presents no further difficulty. However to analyze the finite-element counterpart of the scheme in Section 2, the inverse assumption,

$$
\sup \left\{h^{2}\|\phi\|_{1}^{2} /\|\phi\|^{2}: \phi \in S_{h}, \phi \neq 0\right\}=\alpha<\infty
$$

(with $\alpha$ independent of $h$ ), is required. Under this hypothesis the condition $\alpha k h^{-2} \leqslant$ $\sigma<1$ guarantees an optimal $k^{2}+h^{r}$ rate of convergence in the $L^{2}$ norm.

The analysis of the Euler-Crank-Nicolson predictor-corrector schemes [5] presents no essential novelty.

Full details of these extensions together with lengthier versions of the proofs of the theorems above can be found in [7], which is available from the present author.

Acknowledgement. The author is very thankful to the referee for his constructive remarks.

Departamento de Ecuaciones Funcionales

Facultad de Ciencias

Universidad de Valladolid

Valladolid, Spain

1. D. N. ARnold, J. Douglas, JR. \& V. ThOMEE, "Superconvergence of a finite element approximation to the solution of a Sobolev equation in a single space variable," Math. Comp., v. 36, 1981, pp. 53-63.

2. T. B. Benjamin \& J. E. Feir, "The disintegration of wave trains in deep water. Part 1," J. Fluid. Mech., v. 27, 1967, pp. 417-430.

3. M. Delfour, M. Fortin \& G. PAYNe, "Finite-difference solution of a nonlinear Schroedinger equation," J. Comput. Phys., v. 44, 1981, pp. 277-288.

4. G. Fairweather, Finite Element Galerkin Methods for Differential Equations, Marcel Dekker, New York, 1978.

5. D. F. Griffiths, A. R. Mitchell \& J. Ll. Morris, A Numerical Study of the Nonlinear Schroedinger Equation, Department of Mathematics, University of Dundee, Report NA/52, May 1982.

6. Kuo Pen-Yu \& J. M. SANZ-Serna, "Convergence of methods for the numerical solution of the Korteweg-de Vries equation”, IMA J. Numer. Anal., v. 1, 1981, pp. 215-221.

7. J. C. Lopez-Marcos, Integración Numérica de la Ecuación de Schroedinger no lineal, M.Sc. thesis, University of Valladolid, Spain, 1983.

8. P. D. Richtmyer \& K. W. Morton, Difference Methods for Initial-Value Problems, InterscienceWiley, New York, 1967.

9. J. M. SAnz-Serna \& V. S. Manoranjan, "A method for the integration in time of certain partial differential equations," J. Comput. Phys., v. 52, 1983, pp. 273-289.

10. W. A. Strauss, "The nonlinear Schroedinger equation" in Contemporary Developments in Continuum Mechanics and Partial Differential Equations (G. M. de la Penha and L. A. Medeiros, Eds.), North-Holland, New York, 1978, pp. 452-465.

11. W. Strauss \& L. Vazquez, "Numerical solution of a nonlinear Klein-Gordon equation," $J$. Comput. Phys., v. 28, 1978, pp. 271-278.

12. M. F. WHEELER, “Apriori $L^{2}$ estimates for Galerkin approximation to parabolic partial differential equations," SIAM J. Numer. Anal., v. 10, 1973, pp. 723-759.

13. H. C. Yuen \& W. E. Ferguson, JR., "Relationship between Benjamin-Feir instability and recurrence in the nonlinear Schroedinger equation," Phys. Fluids, v. 21, 1978, pp. 1275-1278.

14. V. E. ZaKharov \& A. B. Shabat, "Exact theory of two-dimensional self-focusing and one-dimensional self-modulation of waves in nonlinear media," Soviet Phys. JETP, v. 34, 1972, pp. 62-69. 